\title{
Blind Equalization Based on Spatial and Temporal Diversity in Block Coded Modulations
}

\author{
Francesc Rey, Meritxell Lamarca, Gregori Vázquez \\ Department of Signal Theory and Communications, \\ UPC Campus Nord-Mòdul D5, c/ Gran Capità s/n, 08034 Barcelona (Spain) \\ E-mail: \{frey,xell,gregori\}@gps.tsc.upc.es
}

\begin{abstract}
Linear block codes can be applied in spatial and/or temporal diversity receivers in order to develop high performance schemes for blind equalization in mobile communications. The proposed technique uses the structure of the encoded transmitted information (with redundancy) to achieve equalization schemes based on a deterministic criterion. Simulations show that the proposed technique is more efficient than other schemes that follow similar equalizer structures. The result is an algorithm that provides the design of blind channel equalizers in low EbNo scenarios.
\end{abstract}

\section{I.INTRODUCTION}

The proposed approach relies on the availability of spatial and/or temporal diversity in digital receivers, which enables the development of blind channel equalization techniques based on a deterministic design criterion. Blind equalizers can suppress the ISI and reduce the impact of the input noise by the knowledge of the transmitted/received data structure or its properties. In such systems no training sequence is needed. For this reason the blind techniques are of practical interest in mobile communications, where the channel is constantly changing and continuous training sequences can not be transmitted.

It is known that if the channel output is oversampled in the time domain and/or in the spatial domain (multi-channel diversity), channel compensation can be performed based on the received signal second-order statistics. A recent study [1] used the Bezout equation to introduce a new blind equalization criterion. Basically, it proposed an algorithm that maximizes the signalto-ISI-plus-noise ratio (SINR) at the equalizer output. Unfortunately, this algorithm offered high reliability only in moderate to high SNR's scenarios but showed stability problems at lower SNR's.

\footnotetext{
* This work has been partially supported by the Spanish Research Council (TIC-95-1022-C05-01) and by ACTS II TSUNAMI-II
}

This paper enhances the results of the method presented in [1], and enables the design of high-performance blind channel equalization schemes in mobile communication scenarios with low EbNo rates. The main idea is to combine the information redundancy introduced by a systematic linear block coding technique with the structure inherent to the 'Transform Modulation' ([2]) transmission techniques to improve the statistical stability of the equalization technique in presence of noise. The result is a more robust scheme that can be applied to TDMA, DS-CDMA systems in frequency selective mobile channels, and OFDM systems in frequency flat fading mobile channels ([3]). Moreover, the proposed solution is available for both spatial and/or temporal diversity front-ends, where it is possible to consider a single-input multiple-output formulation (SIMO) of the transmission system.

In some equalization methods ([4]) the transmitter introduces redundancy that the receiver uses to identify or equalize the channel. Although some additional redundancy is also added in our method, the proposed approach can be considered a blind technique because that redundancy is not introduced to equalize but used to correct detection errors. Thus the reception is done in two steps. In the first step the receiver uses the structure of the encoded transmitted information (with redundancy) to achieve the equalization. In the second step the same redundancy is also used to correct errors. This paper studies the first part of the whole receiver, analyzing the performance of the equalizer using the redundancy symbols. It will be the aim of a later study to choose linear codes defined over the complex field $([5],\{6])$ that, applying the structure presented in this paper, could be used to correct decision errors at the output of the equalizer.

The next section illustrates the scheme and establishes the problem. Section 3 describes the linear block coding characteristics and shows how the structure of the transmitted encoded data can be used in the design of the equalizer. Section 4 applies these results to improve the [1] cost function. Finally section 5 presents some simulation results, where it is possible to see the improvement of the proposed solution. 


\section{PROBLEM STATEMENT}

The proposed approach relies on the availability of spatial and/or temporal diversity in digital receivers, which enables the use of single-input multiple-output formulation (SIMO) of the transmission system. It consists of an algorithm for linear equalization of the received data that is based on a deterministic design criterion.

Figure 1a shows a discrete-time model for a spatial diversity receiver. The same information signal $T[k]$ is transmitted through $B$ diversity branches. It is distorted by different channel responses $C^{i}[k]$ and finally degraded by $A W G N$ terms $W^{i}[k]$.

In a similar way, Figure $1 b$ shows the model for a temporal diversity receiver. The information signal $T[k]$ is transmitted through a channel, which distorts the signal with a channel response $C[k]$ and introduces an AWGN, $W[k]$. The received signal is oversampled, at B samples per symbol, and introduced in $\mathrm{B}$ different branches as in the previous structure.

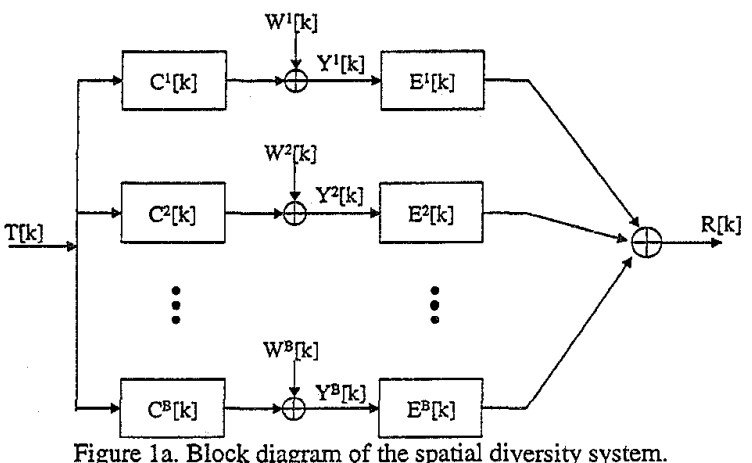

The equalization process for spatial and temporal diversity schemes is similar, and both can be combined into the same receiver system. Their associated equations can be written in the z-transform domain as:

$$
Y^{i}(z)=T(z) C^{i}(z)+W^{i}(z) \quad i=1, \ldots, B
$$

Similar equations can be derived in OFDM signals through frequency-flat fading channels in the time domain (see [1], [7]).

As shown in [1], the equalization process can be designed following a blind criterion. The multiple temporal or spatial diversity branches are combined by means of FIR filters $E^{i}[k]$ to generate an output $R[k]$ :

$$
\begin{aligned}
R(z) & =\sum_{i=1}^{B} Y^{i}(z) E^{\prime}(z)= \\
& =T(z) \sum_{i=1}^{B} C^{\prime}(z) E^{i}(z)+\sum_{i=1}^{B} W^{i}(z) E^{i}(z)
\end{aligned}
$$

The perfect equalization criterion requires $R(z)=T(z)$, and therefore (neglecting the noise effect):

$$
\sum_{i=1}^{B} C^{i}(z) E^{i}(z)=1
$$

The Bezout equation [8] guarantees that the previous equation has solution if and only if the $B$ channel responses have no common zeros, a result well known in the literature ([9]), and therefore (ignoring the noise term):

$$
T(z)=g . c . d .\left\{y^{i}(z)\right\}
$$

A matrix formulation for the method can be found in [10] and is briefly summarized here in order to approach the problem. As shown in [10], equation (2) can be written in matrix notation as:

$$
\underline{R}=\underline{\underline{Y}} \underline{\underline{E}}
$$

where $R$ is the equalizer output vector, $Y$ is a generalized Sylvester matrix with the received data and $\underline{E}$ is the equalizer weight vector. The perfect equalization case in (3) can be written as:

$$
\underline{R}=\left[\begin{array}{l}
\underline{T} \\
\underline{0}
\end{array}\right] \alpha=\left[\begin{array}{l}
Y_{t} \\
\underline{Y_{0}}
\end{array}\right]
$$

where the received data matrix $\underline{Y}$ has been split in two parts, $\underline{T}$ is the transmitted data vector and $\alpha$ is an arbitrary constant. The optimum equalizer coefficients (if the noise term is not considered) can be estimated with:

$$
\underline{\underline{Y_{0}}} \underline{\underline{E}}=\underline{0}
$$

and once the equalizer has been estimated, the received data can be filtered to yield an estimation of the transmitted data:

$$
\hat{\underline{T}}=\underline{Y_{1}} \underline{\underline{E}}
$$

An interesting algorithm, formulated in [1], tries to maximize the signal-to-ISI-plus-noise-ratio (SINR) at the equalizer output, that is:

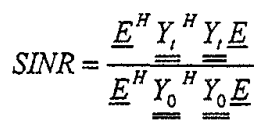

and the equalizer coefficients are designed to maximize the previous quotient.

In high EbNo scenarios the previous cost function performs correctly, but in noisy environments many stability problems with the $Y_{0}$ matrix appear. Hence, the denominator of (9) is critical and new design criteria should be found.

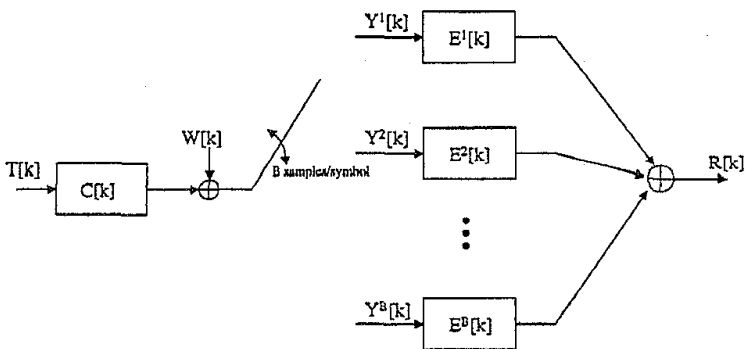

Figure Ib. Block diagram of the temporal diversity system. 


\section{LINEAR BLOCK CODING STRUCTURES}

Equation (10) details the construction of linear block codes using matrix notation. It describes how to encode $k$ symbol data information using the code generator matrix $\underline{G}_{c}$ and obtain the $n$ coded output sequence:

$$
\underline{T^{\prime}}=\underline{\underline{G_{c}}} \underline{\underline{T}}
$$

Considering a systematic code, the encoder matrix becomes:

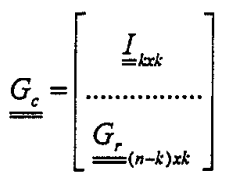

and the code word is divided into two parts. The first k-symbol partition is always identical to the information sequence to be transmitted, while in the second portion each of the (n-k) symbols is a linear combination of the information data according to $G_{r}$ :

$$
\underline{T}^{\prime}=\left[\begin{array}{c}
\underline{T}_{k} \\
\cdots \cdots \cdots \cdots \\
\underline{R}_{(n-k)}
\end{array}\right]
$$

The second step in the coding process applies the parity check matrix $\underline{\underline{C_{c}}}$ over the mobile channel output data. This matrix is defined as:

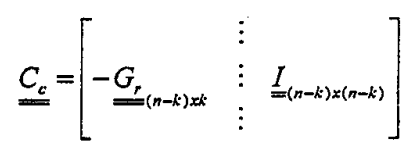

and $\underline{\underline{G_{c}}}$ and $\underline{\underline{C_{c}}}$ matrices are defined so that:

$$
\underline{\underline{C_{c} G_{c}} \underline{\underline{T}}}=\underline{0}
$$

Thus the parity check matrix can detect changes between the transmitted code and the received information. If both sequences are identical, the null vector will be obtained. On the contrary, the effect of the noise and the ISI channel introduces differences in the received sequence, and the product of that sequence with the parity check matrix differs from the null vector.

Because of the construction of $\underline{\underline{G_{c}}}$ and $\underline{\underline{C_{c}}}$ matrices, any full rank matrix can be used as parity check symbol matrix $G_{r}$. Furthermore to ensure that the encoding process maintains constant the symbol energy of all the transmitted symbols we are interested on those transform such that:

$$
\left|g_{1}\right|^{2}=\left|g_{2}\right|^{2}=\ldots=\left|g_{n}\right|^{2}=1
$$

where $\left|g_{i}\right|$ is the norm of the row vectors of $\underline{\underline{G_{c}}}$.

The introduction of the redundancy provides a more rich structure in the transmitted information, which can be used in the design of the equalizer. Previously, we have seen how the parity or redundancy symbols allow the receiver to detect the effects of the ISI and noise, so new constraints can be introduced to find the equalizer weight vector $\underline{E}$.

As in equation (8), if the $\underline{E}$ coefficients equalize correctly the received data, the filter gives a good estimation of the transmitted sequence:

$$
\underline{T^{\prime}}=\underline{\underline{Y_{t}}} \underline{\underline{E}}
$$

hence, applying the check parity matrix to $\underline{T}^{\prime}$ a perfect equalization expression can be found:

$$
\underline{\underline{C_{c}}} \underline{\underline{T^{\prime}}}=\underline{\underline{C_{c}} Y_{t}} \underline{\underline{E}}=\underline{0}
$$

The previous equation can also be seen as a measure of the quality of the equalizer. The closer to zero it is, the better the equalizer performs, whereas any non-null residue in (17) will denote a poor channel equalization.

\section{THE COST FUNCTION IMPROVEMENT}

As it was said before, equation (7) can exhibit poor performance in very low SNR scenarios. In this paper, a new method to design equalization coefficients and to overcome this problem, is proposed. The cost function equation tries to maximize the SINR at the output, and we have previously discussed that if

$$
\underline{\underline{C_{c} Y_{t}}} \underline{\underline{E}}
$$

is close to zero it means that the equalizer is reducing the ISI effect and filtering the additive noise correctly.

According to this consideration the new cost function is formulated as:

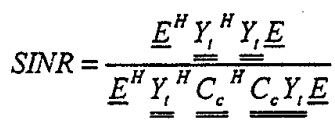

With this improvement the function to be optimized is more robust in presence of AWGN, it only needs the signal submatrix $Y_{t}$, avoiding the use of the residual $Y_{0}$ matrix, which uses to be ill conditioned. Notice that the changes have been done in the denominator, which was the critical part of the quotient:

Equation (19) corresponds to a typical Rayleigh quotient form (see [11]), and therefore the equalizer ( $E$ vector) that maximizes it corresponds to the generalized eigenvector associated with the maximum generalized eigenvalue:

$$
\underline{\underline{Y_{t}^{H}}} \underline{\underline{Y_{t}}} \underline{\underline{E}}=\lambda_{\max } \underline{\underline{Y_{t}^{H}}} \underline{\underline{\underline{C_{c}}}}{ }^{H} \underline{\underline{C_{c} Y_{t}}} \underline{\underline{E}}
$$

The equalization performance can be also improved if a delay is allowed in $R[k]$, and the best equalizer is selected as that one which yields the greatest $\lambda_{\max }$.

Finally it is also worth remarking that the computational load of the solution does not increase if adequate matrices are selected for $G_{r}$. In our study sub-Hadamard matrices have been chosen. 


\section{SIMULATIONS}

Figures 2 to 6 compare the performance of the cost function in [1] with the new cost function proposed in this paper.

The simulations display the percentage of realizations (1000 were averaged) for which the equalizer output EbNo was higher than the value indicated in the $\mathrm{x}$-axis. In all cases the transmitted information consisted of 118 QPSK data symbols and 10-redundancy symbols generated with a sub-Hadamard matrix $G_{r}$. Each branch equalizer had four coefficients and four branches $(B=4)$ were simulated.

Figures 2 and 3 show the performance of the algorithm using a TDMA burst transmission over a frequency selective channel with spatial diversity (Figurela). The four stationary channel responses were:

$$
\begin{aligned}
& C^{1}(z)=(1+j)+(-0.1-0.2 j) z^{-1}+0.4 z^{-2}+z^{-3}+0.5 z^{-4} \\
& C^{2}(z)=0.1+2 z^{-1}+4 j z^{-2}+0.2 z^{-3}+z^{-4} \\
& C^{3}(z)=0.1 j+z^{-1}-0.4 j z^{-2}+0.2 z^{-3}-0.5 z^{-4} \\
& C^{4}(z)=(1+0.8 j)-2 j z^{-1}-0.4 j z^{-2}+0.2 z^{-3}+(1-0.5 j) z^{-4}
\end{aligned}
$$

Notice that the four channels have no common zeros. In order to consider a difficult mobile scenario, the four channels have been selected with high attenuation in certain frequencies (in special channels 1 and 3 ), with some close zeros and with a non-minimum phase behavior.

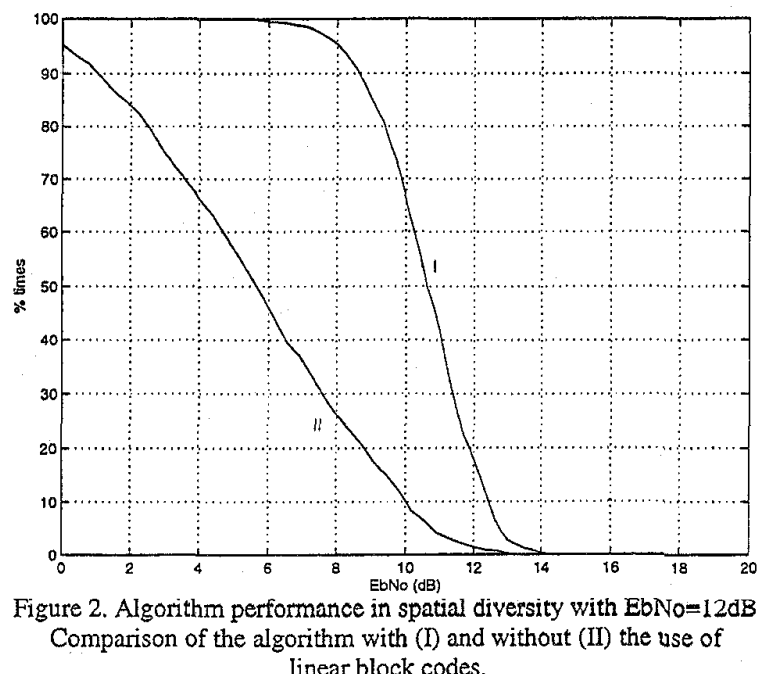

In Figure2 the channel EbNo is $12 \mathrm{~dB}$, while Figure 3 illustrates the simulation with $E b N o=15 \mathrm{~dB}$. In both cases the performance of the improved cost function (I) is higher than the performance of the algorithm without linear block codes (II). Notice that the new method guarantees that with a EbNo higher than $12 \mathrm{~dB}$, more than the $95 \%$ of times the output EbNo is over $7 \mathrm{~dB}$, which represents a $B E R \geq 10^{-3}$.

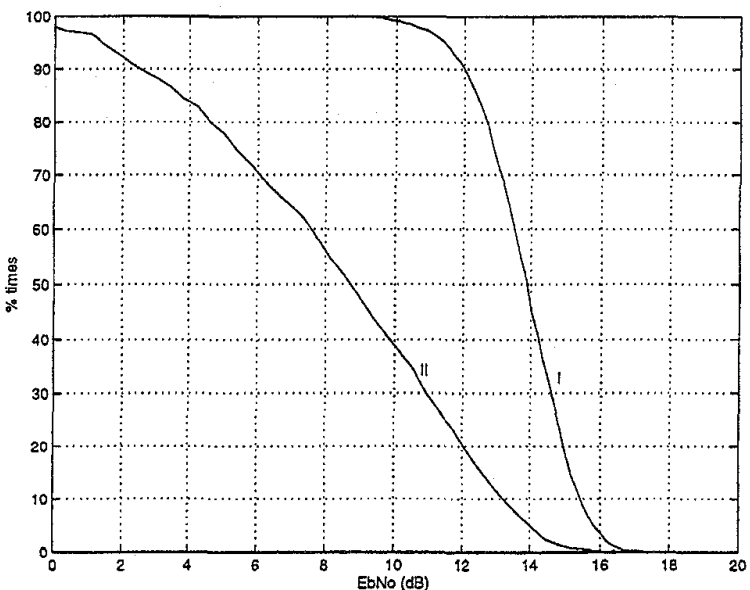

Figure 3. Algorithm performance in spatial diversity with $\mathrm{EbNo}=15 \mathrm{~dB}$ Comparison of the algorithm with (I) and without (II) the use of linear block codes.

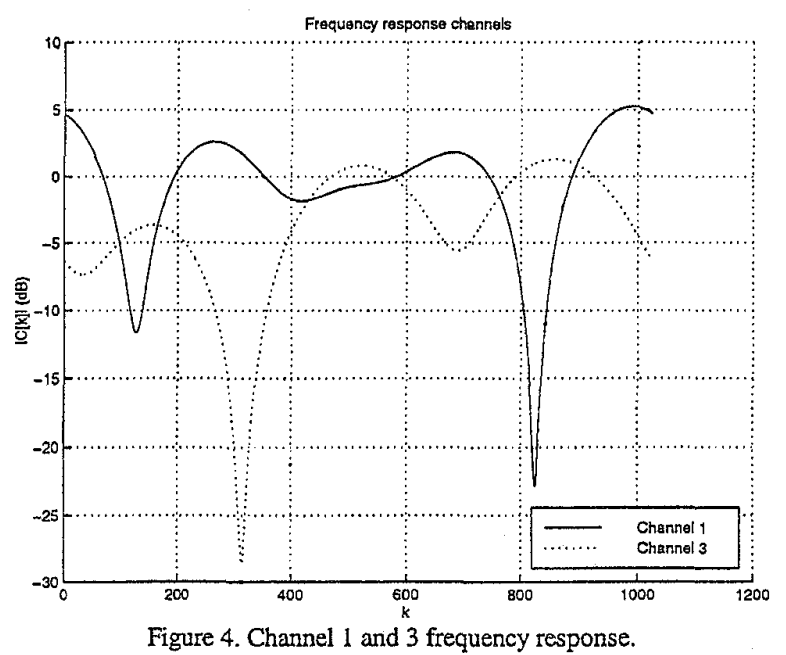

Figures 5 and 6 show the performance of the algorithm in a TDMA burst transmission with temporal-diversity (Figure 1b). Two different non-minimum phase channels were simulated: $C^{1}[z] ; C^{3}[z]$. Their frequency responses are illustrated in Figure. 4. Figure 5 illustrates the simulation with $E b N o=12 \mathrm{~dB}$, and Figure 6 with $E b N o=15 \mathrm{~dB}$. The dashed line curves are related to channel 3 , and the solid curves to channel 1 . As in the previous case I represents the results using linear block codes and II the results without them. Notice that the performance with spatial diversity is better than with temporal diversity. Two reasons can justify that. First, the spatial diversity uses $B$ antennas, and so the maximum output EbNo could be:

$$
\max \left\{(E b N O)_{\text {out }}\right\}=(E b N O)_{i n}+10 \log (B)
$$

meanwhile in temporal diversity:

$$
\max \left\{(E b N o)_{\text {out }}\right\}=(E b N o)_{\text {in }}
$$

Second, when the Nyquist pulse is oversampled (in temporal diversity), additional ISI is introduced into the receiver, causing the performance of the system to be reduced. 


\section{VI.CONCLUSIONS}

In this paper linear block codes in the complex field have been introduced for blind equalization of mobile channels in spatial and temporal diversity receivers.

The proposed criterion, combining a blind equalization technique ([1]) with the redundancy introduced by a systematic linear code, can be applied over TDMA structures with frequency selective mobile channels, DS-CDMA systems and OFDM modulation with frequency flat fading channels.

Spatial and temporal diversity receivers over TDMA structures, in frequency selective mobile channels, have been considered. The results show the performance improvement of this new equalization method over the previous scheme presented in [1].

Better results that those presented in the current paper can be achieved introducing codes defined over the complex fields $([5],[6])$ that, following the structure presented in section 3 , could be used to correct most of the errors at the output of the equalizer.

\section{REFERENCES}

[1] M. Lamarca, G. Vázquez, 'Diversity techniques for blind channel equalization in mobile communications', Proceedings. of PIMRC'97, pag. 1079-1083, Helsinki (Finland), Sept. 1997.

[2] M. Lamarca, G. Vázquez, 'Transform modulations in mobile communications', Proc. of PIMRC'97, pag 462466, Helsinki (Finland), Sept. 1997.

[3] R Steele, Mobile Radio Communications, Pentech Press.

[4] A. Scaglione, G.B. Giannakis, S. Barbarossa 'Selfrecovering multirate equalizers using redundant filterbank precoders", Proceedings of ICASSP'98 Seattle May 1998.

[5] T.G. Marshall, 'Coding of real-number sequences for error correction: a digital signal processing problem' IEEE Journal on Selected Areas in Communications, Vol.2, March 1984.

[6] R.E. Blahut, 'Algebraic fields, signal processing, and error control', Proceedings of the IEEE, Vol.73, no 5, May 85.

[7] M. Lamarca, G. Vázquez, 'Channel estimation for transform modulations in mobile communications', Proceedings of EUSIPCO'96, Trieste (Italy), Sept. 1996.

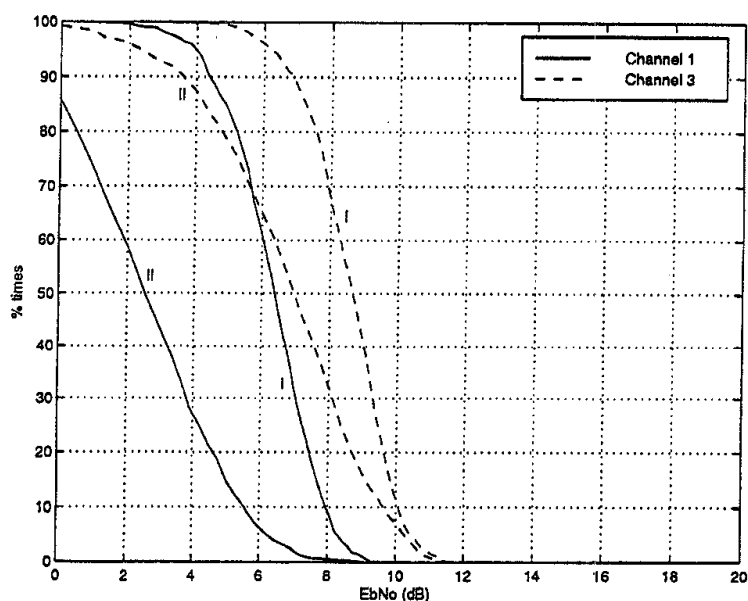

Figure 5. Algorithm performance in temporal diversity with $E b N o=12 \mathrm{~dB}$ through different channels. Comparison of the algorithm with (I) and without (II) the use of linear block codes.

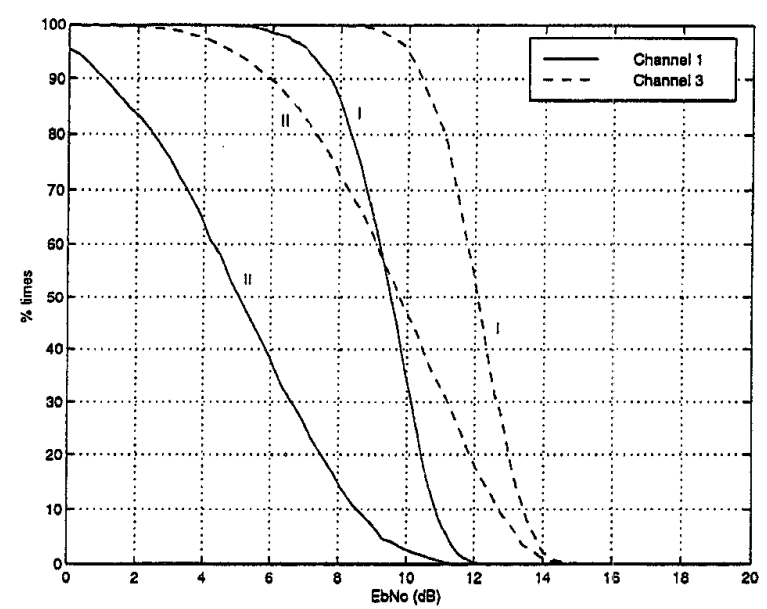

Figure 6. Algorithm performance in temporal diversity with $\mathrm{EbNo}=15 \mathrm{~dB}$, through different channels. Comparison of the algorithm with (I) and without (II) the use of linear block codes.

[8] T. Kailath, Linear Systems, Prentice-Hall, 1980.

[9] Y.lil, Z.Ding, 'Blind channel identification based on second order cyclostacionary statistics', Proceedings. of ICASSP'93 Minneapolis (USA)

[10] M. Lamarca, G. Vázquez, 'Multichannel receivers for OFDM and TDM in mobile communications', Proc. of ICASSP '97, Munich (Germany), April. 1997.

[11] G.H. Golub, C.F. Van Loan, Matrix Computations, Johns Hopkins Univ. Press, 1989. 